\title{
Quintessence Ghost Dark Energy Model
}

\author{
Ahamd Sheykhi ${ }^{1,2 *}$ and Ali Bagheri ${ }^{1}$ \\ ${ }^{1}$ Department of Physics, Shahid Bahonar University, P.O. Box 76175, Kerman, Iran \\ ${ }^{2}$ Research Institute for Astronomy and Astrophysics of Maragha (RIAAM), Maragha, Iran
}

\begin{abstract}
A so called "ghost dark energy" was recently proposed to explain the present acceleration of the universe expansion. The energy density of ghost dark energy, which originates from Veneziano ghost of $\mathrm{QCD}$, is proportional to the Hubble parameter, $\rho_{D}=\alpha H$, where $\alpha$ is a constant which is related to the QCD mass scale. In this paper, we establish the correspondence between ghost dark energy and quintessence scalar field energy density. This connection allows us to reconstruct the potential and the dynamics of the quintessence scalar field according to the evolution of ghost energy density.
\end{abstract}

\section{INTRODUCTION}

A wide range of cosmological observations, direct and indirect, provide an impressive evidence in favor of the present acceleration of the cosmic expansion. To explain this acceleration, in the context of standard cosmology, we need an anti gravity fluid with negative pressure, usually dubbed "dark energy" in the literature. The first and simple candidate for dark energy is the cosmological constant with equation of state parameter $w=-1$ which is located at the central position among dark energy models both in theoretical investigation and in data analysis [1]. However, there are several difficulties with cosmological constant. For example, it suffers the so-called fine-tuning and cosmic coincidence problems. Besides, the origin of it is still a much source of doubt. Furthermore, the accurate data analysis, show that the time varying dark energy gives a better fit than a cosmological constant and in particular, $w$ can cross -1 around $z=0.2$ from above to below [2]. Although the galaxy cluster gas mass fraction data do not support the time-varying $w$ [3] , an overwhelming flood of papers has appeared which attempt to understand the $w=-1$ crossing. Among them are a negative kinetic scalar field and a normal scalar field [4], or a single scalar field model [5], interacting holographic [6] and interacting agegraphic [7] dark energy models. Other studies on the $w=-1$ crossing [8] and dark energy models have been carried out in [9]. For a recent review on dark energy models see [10]. It is worthy to note that in most of these dark energy models, the accelerated expansion are explained by introducing new degree(s) of freedom or by modifying the underlying theory of gravity.

Recently a very interesting suggestion on the origin of a dark energy is made, without introducing new degrees of freedom beyond what are already known, with the dark energy of just the right magnitude to give the observed expansion [11, 12]. In this proposal, it is claimed that the cosmological constant arises from the contribution of the ghost fields which are supposed to be present in the low-energy effective theory of QCD [13 17]. The ghosts are required to exist for the resolution of the $U(1)$ problem, but are completely decoupled from the physical sector [17]. The above claim is that the ghosts are decoupled from the physical states and make no contribution in the flat Minkowski space, but once they are in the curved space or time-dependent background, the cancelation of their contribution to the vacuum energy is off-set, leaving a small energy density $\rho \sim H \Lambda_{Q C D}^{3}$, where $H$ is the Hubble parameter and $\Lambda_{Q C D}$ is the QCD mass scale of order a $100 \mathrm{MeV}$. With $H \sim 10^{-33} \mathrm{eV}$, this gives the right magnitude $\sim\left(3 \times 10^{-3} \mathrm{eV}\right)^{4}$ for the observed dark energy density. This numerical coincidence is remarkable and also means that this model gets rid of fine tuning problem [11, 12]. The advantages of this new model compared to other dark energy models is that it is totally embedded in standard model and general relativity, one needs not to introduce any new parameter, new degree of freedom or to modify gravity. The dynamical behavior of the ghost dark energy (GDE) model in flat [18] and non flat [19] universe have been studied in ample details.

On the other side, the scalar field model can be regarded as an effective description of an underlying dark energy theory. Scalar fields naturally arise in particle physics including supersymmetric field theories and string/M theory. Therefore, scalar field is expected to reveal the dynamical mechanism and the nature of dark energy. However, although fundamental theories such as string/M theory do provide a number of possible candidates for scalar fields, they do not predict its potential $V(\phi)$ uniquely. Consequently, it is meaningful to reconstruct the potential $V(\phi)$ from some dark energy models possessing some significant features of the quantum gravity theory, such as holographic and agegraphic dark energy models. In the framework of holographic and agegraphic dark energy models, the studies on the reconstruction of the quintessence potential $V(\phi)$ have been carried out in [20] and [21], respectively. Till now, quintessence reconstruction of ghost energy density has not been done.

\footnotetext{
*sheykhi@mail.uk.ac.ir
} 
In this paper we are interested in that if we assume the GDE scenario as the underlying theory of dark energy, how the low-energy effective scalar-field model can be used to describe it. In this direction, we can establish the correspondence between the GDE and quintessence scalar field, and describe GDE in this case effectively by making use of quintessence. We shall reconstruct the quintessence potential and the dynamics of the scalar field in the light of the GDE.

\section{QUINTESSENCE GHOST DARK ENERGY}

We assume the GDE is accommodated in a flat Friedmann-Robertson-Walker (FRW) which its dynamics is governed by the Friedmann equation

$$
H^{2}=\frac{1}{3 M_{p}^{2}}\left(\rho_{m}+\rho_{D}\right),
$$

where $\rho_{m}$ and $\rho_{D}$ are the energy densities of pressureless matter and GDE, respectively. We define the dimensionless density parameters as

$$
\Omega_{m}=\frac{\rho_{m}}{\rho_{\mathrm{cr}}}, \quad \Omega_{D}=\frac{\rho_{D}}{\rho_{\mathrm{cr}}}
$$

where the critical energy density is $\rho_{\mathrm{cr}}=3 H^{2} M_{p}^{2}$. Thus, the Friedmann equation can be rewritten as

$$
\Omega_{m}+\Omega_{D}=1 \text {. }
$$

The conservation equations read

$$
\begin{aligned}
\dot{\rho}_{m}+3 H \rho_{m} & =0, \\
\dot{\rho}_{D}+3 H \rho_{D}\left(1+w_{D}\right) & =0 .
\end{aligned}
$$

The ghost energy density is proportional to the Hubble parameter [12, 18]

$$
\rho_{D}=\alpha H
$$

where $\alpha$ is a constant of order $\Lambda_{\mathrm{QCD}}^{3}$ and $\Lambda_{\mathrm{QCD}} \sim 100 \mathrm{MeV}$ is QCD mass scale. Taking the time derivative of relation (6) and using Friedmann equation (11) we find

$$
\dot{\rho}_{D}=-\frac{\alpha}{2 M_{p}^{2}} \rho_{D}\left(1+u+w_{D}\right) .
$$

where $u=\rho_{m} / \rho_{D}$ is the energy density ratio. Inserting this relation in continuity equation (5) and using Eq. (3) we find

$$
w_{D}=-\frac{1}{2-\Omega_{D}} .
$$

At the early time where $\Omega_{D} \ll 1$ we have $w_{D}=-1 / 2$, while at the late time where $\Omega_{D} \rightarrow 1$ the GDE mimics a cosmological constant, namely $w_{D}=-1$. In figure 1 we have plotted the evolution of $w_{D}$ versus scale factor $a$. From this figure we see that $w_{D}$ of the GDE model cannot cross the phantom divide and the universe has a de Sitter phase at late time.

Now we are in a position to establish the correspondence between GDE and quintessence scaler field. To do this, we assume the quintessence scalar field model of dark energy is the effective underlying theory. The energy density and pressure of the quintessence scalar field are given by

$$
\begin{aligned}
\rho_{\phi} & =\frac{1}{2} \dot{\phi}^{2}+V(\phi), \\
p_{\phi} & =\frac{1}{2} \dot{\phi}^{2}-V(\phi) .
\end{aligned}
$$

Thus the potential and the kinetic energy term can be written as

$$
\begin{aligned}
& V(\phi)=\frac{1-w_{\phi}}{2} \rho_{\phi}, \\
& \dot{\phi}^{2}=\left(1+w_{\phi}\right) \rho_{\phi} .
\end{aligned}
$$




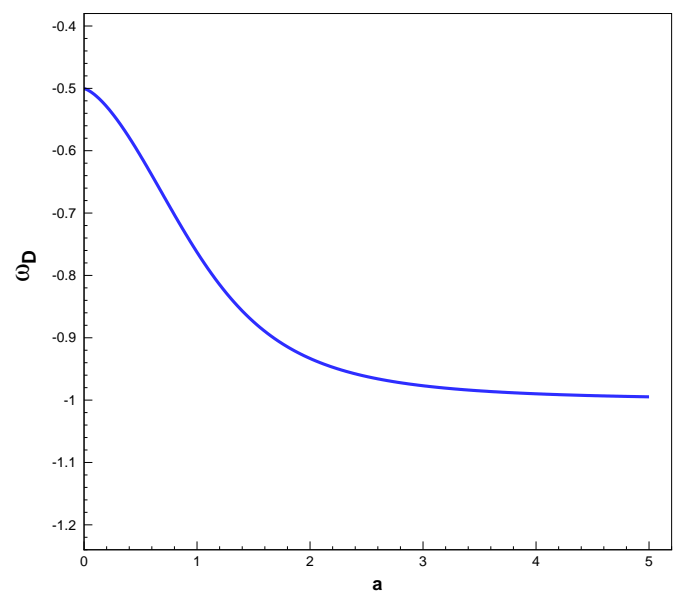

FIG. 1: The evolution of $w_{D}$ for GDE.

In order to implement the correspondence between GDE and quintessence scaler field, we identify $\rho_{\phi}=\rho_{D}$ and $w_{\phi}=w_{D}$. Using Eqs. (6) and (8) as well as relation $\dot{\phi}=H \frac{d \phi}{d \ln a}$ we obtain the scalar potential and the dynamics of scalar field as

$$
\begin{aligned}
V(\phi) & =\frac{\alpha^{2}}{6 M_{p}^{2}} \times \frac{3-\Omega_{D}}{\Omega_{D}\left(2-\Omega_{D}\right)}, \\
\frac{d \phi}{d \ln a} & =\sqrt{3} M_{p} \sqrt{\frac{\Omega_{D}\left(1-\Omega_{D}\right)}{2-\Omega_{D}}} .
\end{aligned}
$$

Integrating yields

$$
\phi(a)-\phi\left(a_{0}\right)=\sqrt{3} M_{p} \int_{a_{0}}^{a} \frac{d a}{a} \sqrt{\frac{\Omega_{D}\left(1-\Omega_{D}\right)}{2-\Omega_{D}}},
$$

where we have set $a_{0}=1$ for the present value of the scale factor. The analytical form of the potential in terms of the ghost quintessence field cannot be determined due to the complexity of the equations involved. However, we can obtain it numerically. The reconstructed quintessence potential $V(\phi)$ and the evolutionary form of the field are plotted in Figs. 2 and 3, where we have taken $\phi\left(a_{0}=1\right)=0$ for simplicity. From figure 2 we can see the dynamics of the scalar field explicitly. Obviously, the scalar field $\phi$ rolls down the potential with the kinetic energy $\dot{\phi}^{2}$ gradually decreasing. In other words, the amplitude of $\phi$ decreases with time in the past.

\section{INTERACTING QUINTESSENCE GHOST DARK ENERGY}

Next we generalize our discussion to the interacting case. Although at this point the interaction may look purely phenomenological but different Lagrangians have been proposed in support of it (see [22] and references therein). Besides, in the absence of a symmetry that forbids the interaction there is nothing, in principle, against it. In addition, given the unknown nature of both dark energy and dark matter, which are two major contents of the universe, one might argue that an entirely independent behavior of dark energy is very special [23, 24]. Thus, microphysics seems to allow enough room for the coupling; however, this point is not fully settled and should be further investigated. The difficulty lies, among other things, in that the very nature of both dark energy and dark matter remains unknown whence the detailed form of the coupling cannot be elucidated at this stage. Since we consider the interaction between dark matter and dark energy, $\rho_{m}$ and $\rho_{D}$ do not conserve separately; they must rather enter the energy balances [24]

$$
\begin{aligned}
\dot{\rho}_{m}+3 H \rho_{m} & =Q, \\
\dot{\rho}_{D}+3 H \rho_{D}\left(1+w_{D}\right) & =-Q,
\end{aligned}
$$




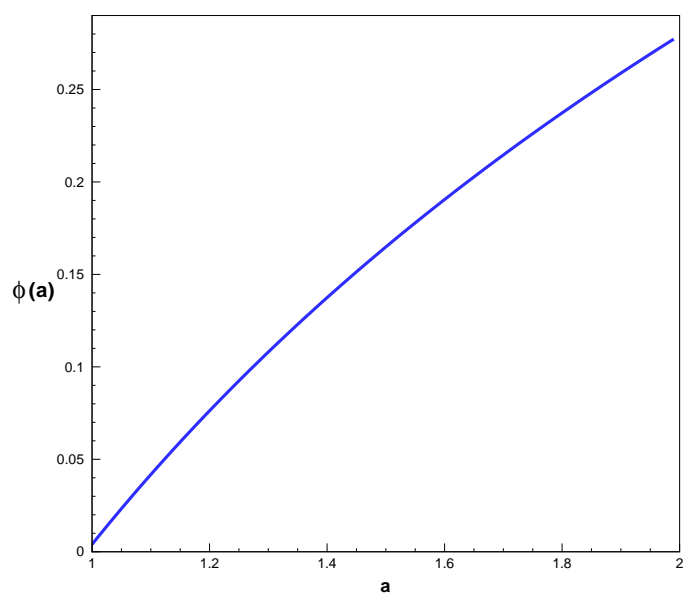

FIG. 2: The evolution of the scalar field $\phi(a)$ for quintessence GDE, where $\phi$ is in unit of $\sqrt{3} M_{p}$.

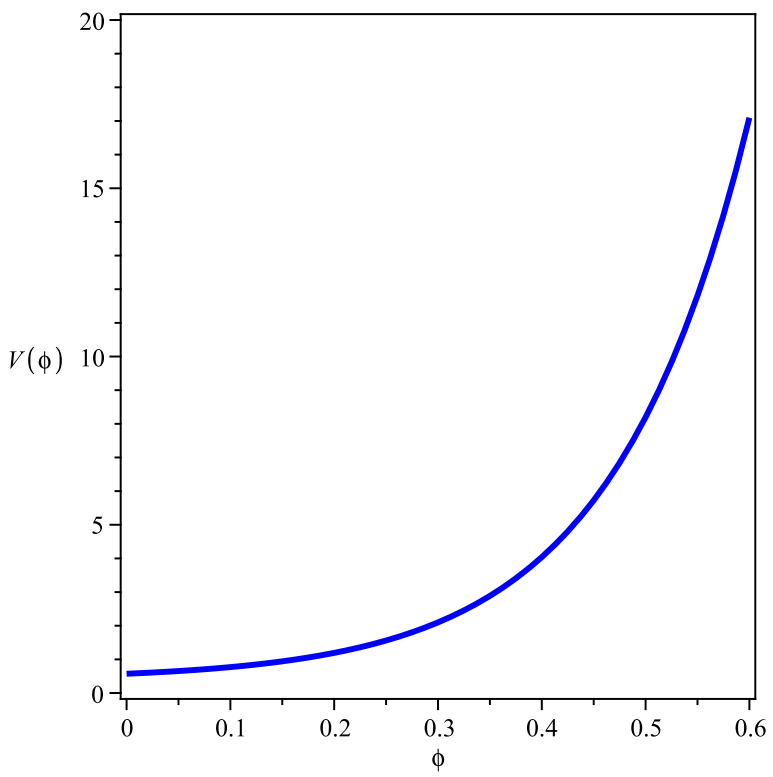

FIG. 3: The reconstructed potential $V(\phi)$ for quintessence GDE, where $V(\phi)$ is in unit of $\alpha^{2} / 6 M_{p}^{2}$.

where $Q$ represents the interaction term and we take it as

$$
Q=3 b^{2} H\left(\rho_{m}+\rho_{D}\right)=3 b^{2} H \rho_{D}(1+u) .
$$

with $b^{2}$ being a coupling constant. Inserting Eqs. (7) and (18) in Eq. (17) we find

$$
w_{D}=-\frac{1}{2-\Omega_{D}}\left(1+\frac{2 b^{2}}{\Omega_{D}}\right) .
$$

One can easily check that in the late time where $\Omega_{D} \rightarrow 1$, the equation of state parameter of interacting GDE necessary crosses the phantom line, namely, $w_{D}=-\left(1+2 b^{2}\right)<-1$ independent of the value of coupling constant $b^{2}$. For the present time with taking $\Omega_{D}=0.72$, the phantom crossing can be achieved provided $b^{2}>0.1$ which is consistent with recent observations [23]. It is worth mentioning that the continuity equations (16) and (17) imply 
that the interaction term should be a function of a quantity with units of inverse of time (a first and natural choice can be the Hubble factor $H$ ) multiplied with the energy density. Therefore, the interaction term could be in any of the following forms: (i) $Q \propto H \rho_{D}$, (ii) $Q \propto H \rho_{m}$, or (iii) $Q \propto H\left(\rho_{m}+\rho_{D}\right)$. We can present the above three choices in one expression as $Q=\Gamma \rho_{D}$, where

$$
\begin{array}{ll}
\Gamma=3 b^{2} H & \text { for } Q \propto H \rho_{D}, \\
\Gamma=3 b^{2} H u & \text { for } Q \propto H \rho_{m}, \\
\Gamma=3 b^{2} H(1+u) & \text { for } Q \propto H\left(\rho_{m}+\rho_{D}\right),
\end{array}
$$

It should be noted that the ideal interaction term must be motivated from the theory of quantum gravity. In the absence of such a theory, we rely on pure dimensional basis for choosing an interaction $Q$. To be more general in this work we choose expression (iii) for the interaction term. The coupling $b^{2}$ is taken in the range [0,1] [25]. Note that if $b^{2}=0$ then it represents the noninteracting case while $b^{2}=1$ yields complete transfer of energy from dark energy to matter $(Q>0)$. Although in principle there is now reason to take $Q>0$ and one may take $Q<0$ which means that dark matter transfers to dark energy, however, as we will see below this is not the case. It is easy to show that for $Q<0$, Eq. (19) becomes

$$
w_{D}=-\frac{1}{2-\Omega_{D}}\left(1-\frac{2 b^{2}}{\Omega_{D}}\right) .
$$

In the late time where $\Omega_{D} \rightarrow 1$, we have $w_{D}=-\left(1-2 b^{2}\right)$, which for $b^{2}>1 / 3$ leads to $w_{D}>-1 / 3$. This implies that in the late time where dark energy dominates we have no acceleration at least for some value of coupling parameter. For the present time if we take $\Omega_{D}=0.72$, from Eq. (21) we have $w_{D}=-0.78+2.2 b^{2}$. Again for $b^{2}>0.20$ we have $w_{D}>-1 / 3$ for the present time. This means that universe is in deceleration phase at the present time which is ruled out by recent observations.

The behaviour of the equation of state parameter of interacting GDE is shown in figure 4 for different value of the coupling parameter. In the presence of interaction, the evolution of GDE is governed by the following equation [19]

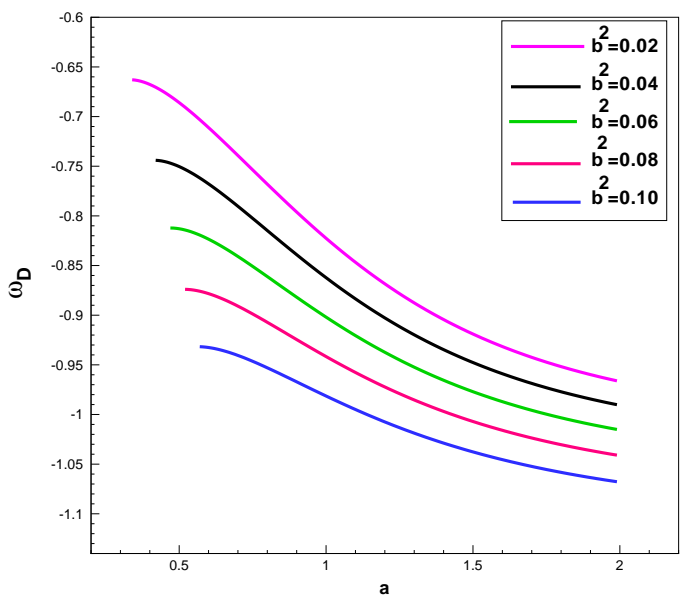

FIG. 4: The evolution of $w_{D}$ for interacting GDE.

$$
\frac{d \Omega_{D}}{d \ln a}=\frac{3}{2} \Omega_{D}\left[1-\frac{\Omega_{D}}{2-\Omega_{D}}\left(1+\frac{2 b^{2}}{\Omega_{D}}\right)\right] .
$$

Fig. 5 shows that at the early time $\Omega_{D} \rightarrow 0$ while at the late time $\Omega_{D} \rightarrow 1$, that is the ghost dark energy dominates as expected. Now we implement a connection between interacting GDE and quintessence scalar field. In this case the potential and scalar field are obtained as

$$
\begin{aligned}
V(\phi) & =\frac{\alpha^{2}}{6 M_{p}^{2}} \times \frac{1}{\Omega_{D}\left(2-\Omega_{D}\right)}\left(3-\Omega_{D}+\frac{2 b^{2}}{\Omega_{D}}\right), \\
\frac{d \phi}{d \ln a} & =\sqrt{3} M_{p} \sqrt{\frac{\Omega_{D}}{2-\Omega_{D}}\left(1-\Omega_{D}-\frac{2 b^{2}}{\Omega_{D}}\right)} .
\end{aligned}
$$




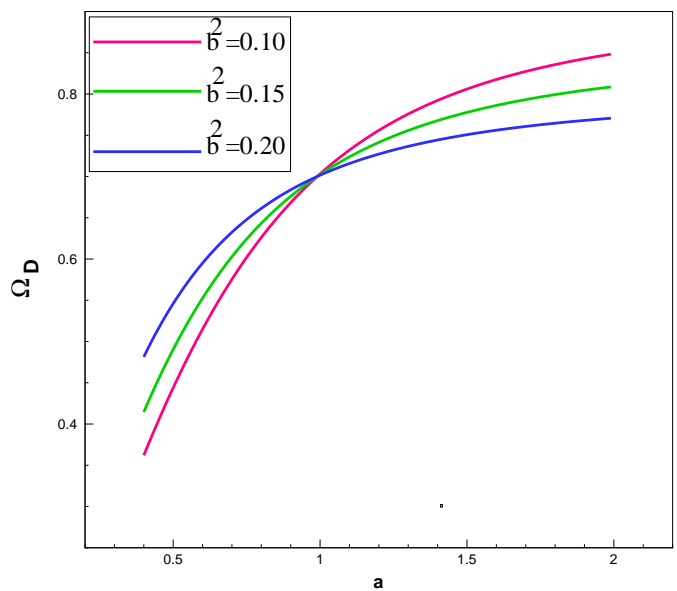

FIG. 5: The evolution of $\Omega_{D}$ for interacting ghost dark energy, where we take $\Omega_{D 0}=0.72$.

Finally we obtain the evolutionary form of the field by integrating the above equation. The result is

$$
\phi(a)-\phi\left(a_{0}\right)=\sqrt{3} M_{p} \int_{a_{0}}^{a} \frac{d a}{a} \sqrt{\frac{\Omega_{D}}{2-\Omega_{D}}\left(1-\Omega_{D}-\frac{2 b^{2}}{\Omega_{D}}\right)},
$$

where $\Omega_{D}$ is now given by Eq. (22). The reconstructed quintessence potential $V(\phi)$ and the evolutionary form of the field are plotted in Figs. 6 and 7, where again we have taken $\phi\left(a_{0}=1\right)=0$ for the present time. Selected curves are plotted for different value of the coupling parameter $b^{2}$. From these figures we find out that $\phi$ increases with time while the potential $V(\phi)$ becomes steeper with increasing $b^{2}$.

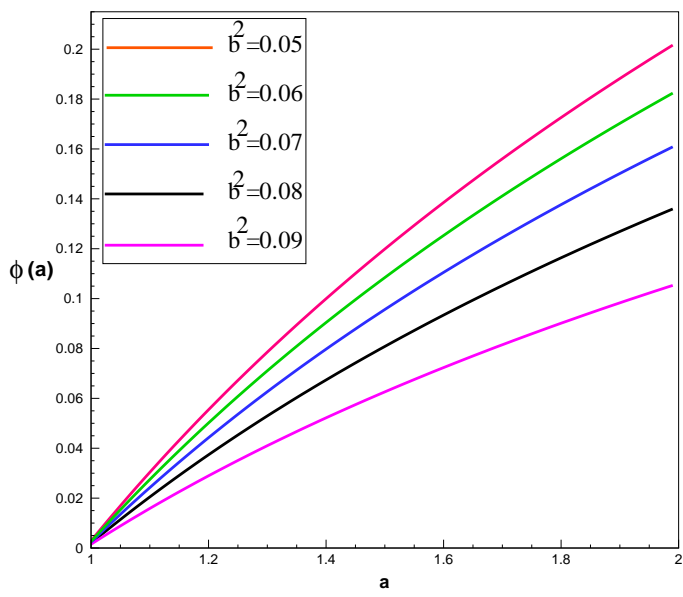

FIG. 6: The evolutionary form of the scalar field $\phi(a)$ for interacting quintessence GDE, where $\phi$ is in unit of $\sqrt{3} M_{p}$. 


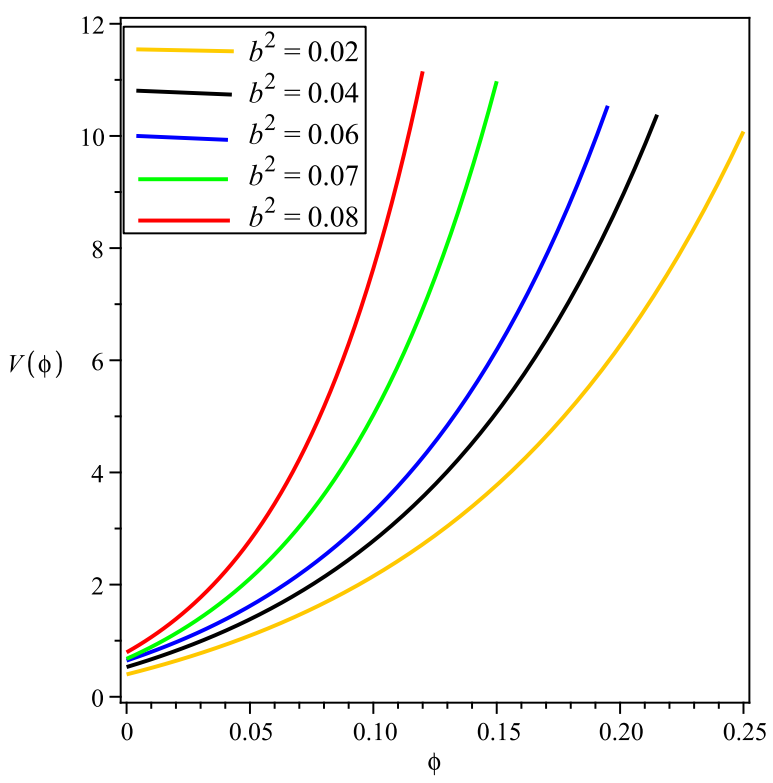

FIG. 7: The reconstructed potential $V(\phi)$ for interacting quintessence GDE, where $V(\phi)$ is in unit of $\left(\alpha^{2} / 6 M_{p}^{2}\right)$.

\section{CONCLUSION}

Considering the quintessence scalar field dark energy model as an effective description of the underlying theory of dark energy, and assuming the ghost vacuum energy scenario as pointing in the same direction, it is interesting to study how the quintessence scalar field model can be used to describe the ghost energy density. The quintessence scalar field is specified to an ordinary scalar field minimally coupled to gravity, namely the canonical scalar field. It is remarkable that the resulting model with the reconstructed potential is the unique canonical single-scalar model that can reproduce the GDE evolution of the universe. In this paper, we established a connection between the GDE scenario and the quintessence scalar-field model. The GDE model is a new attempt to explain the origin of dark energy within the framework of Veneziano ghost of QCD [12]. If we regard the quintessence scalar-field model as an effective description of GDE, we should be capable of using the scalar-field model to mimic the evolving behavior of the dynamical ghost energy and reconstructing this scalar-field model according to the evolutionary behavior of GDE. With this strategy, we reconstructed the potential of the ghost quintessence and the dynamics of the field according to the evolution of ghost energy density.

Finally we would like to mention that the aforementioned discussion in this paper can be easily generalized to other non-canonical scalar fields, such as K-essence and tachyon. It can also be extended to the non-flat FRW universe.

\section{Acknowledgments}

This work has been supported by Research Institute for Astronomy and Astrophysics of Maragha, Iran.

[1] S. Weinberg, Rev. Mod. Phys. 61, 1 (1989);

N. Straumann, arXiv:astro-ph/0203330.

T. Padmanabhan, Classical Quantum Gravity 22, L107 (2005).

[2] U. Alam, V. Sahni and A. Starobinsky, JCAP 0406 (2004) 008;

Y.G. Gong, Class. Quant. Grav. 22 (2005) 2121;

U. Alam, V. Sahni, T. Saini and A. Starobinsky, Mon. Not. Roy. Astron. Soc. 354, (2004) 275;

T. Choudhury and T. Padmanabhan, Astron. Astrophys. 429, (2005) 807.

[3] G. Chen and B. Ratra, Astrophys. J. 612, (2004) L1. 
[4] B. Feng, X. L.Wang and X. M. Zhang, Phys. Lett. B 607 (2005) 35;

W. Hu, Phys. Rev. D 71, (2005) 047301;

Z.K. Guo, Y.S. Piao, X.M. Zhang and Y.Z. Zhang, Phys. Lett. B 608, (2005) 177.

[5] M.Z. Li, B. Feng and X.M. Zhang, JCAP 0512 (2005) 002.

[6] B. Wang, Y. Gong, E. Abdalla, Phys. Lett. B 624, (2006) 141;

B. Wang, C. Y. Lin, E. Abdalla, Phys. Lett. B 637, (2006) 357;

B. Wang, J.D. Zang, C.Y. Lin, E. Abdalla and S. Micheletti, Nucl. Phys. B 778 (2007) 69;

A. Sheykhi, Phys Lett B 681 (2009) 205;

A. Sheykhi, Class. Quantum Grav. 27 (2010) 025007.

[7] H. Wei and R. G. Cai, Phys. Lett. B 660 (2008) 113;

H. Wei and R. G. Cai, Eur. Phys. J. C 59 (2009) 99;

A. Sheykhi, Phys. Lett. B 680 (2009) 113;

A. Sheykhi, Phys. Rev. D 81 (2010) 023525;

A. Sheykhi, Phys. Lett. B 682 (2010) 329.

[8] S. Nojiri and S.D. Odintsov, Phys. Lett. B 562, (2003) 147;

S. Nojiri and S. D. Odintsov, Phys. Rev. D 70 (2004) 103522;

S. Nojiri, S.D. Odintsov and S. Tsujikawa, Phys. Rev. D 71 (2005) 063004;

A. Vikman, Phys. Rev. D 71, (2005) 023515;

A. Anisimov, E. Babichev and A. Vikman, JCAP 0506, (2005) 006;

A. Sheykhi, B. Wang and N. Riazi, Phys. Rev. D 75, 123513 (2007).

[9] S. Nojiri, S.D. Odintsov, Phys. Lett. B 637 (2006) 139;

S. Nojiri, S.D. Odintsov, Int. J. Geom. Meth. Mod. Phys. 4 (2007) 115;

S. Nojiri, S.D. Odintsov, Phys. Lett. B 659 (2008) 821;

S. Nojiri, S.D. Odintsov, P. V. Tretyakov, Prog. Theor. Phys. Suppl. 172 (2008) 81.

[10] E. J. Copeland, M. Sami and S. Tsujikawa, Int. J. Mod. Phys. D 15, 1753 (2006).

[11] F. R. Urban and A. R. Zhitnitsky, Phys. Lett. B 688 (2010) 9 ;

F. R. Urban and A. R. Zhitnitsky, Phys. Rev. D 80 (2009) 063001;

F. R. Urban and A. R. Zhitnitsky, JCAP 0909 (2009) 018;

F. R. Urban and A. R. Zhitnitsky, Nucl. Phys. B 835 (2010) 135.

[12] N. Ohta, Phys. Lett. B 695 (2011) 41,

[13] E. Witten, Nucl. Phys. B 156 (1979) 269.

[14] G. Veneziano, Nucl. Phys. B 159 (1979) 213.

[15] C. Rosenzweig, J. Schechter and C. G. Trahern, Phys. Rev. D 21 (1980) 3388.

[16] P. Nath and R. L. Arnowitt, Phys. Rev. D 23 (1981) 473.

[17] K. Kawarabayashi and N. Ohta, Nucl. Phys. B 175 (1980) 477; Prog. Theor. Phys. 66 (1981) 1789; N. Ohta, Prog. Theor. Phys. 66 (1981) 1408.

[18] R.G. Cai, Z.L. Tuo, H.B. Zhang, arXiv:1011.3212

[19] A. Sheykhi, M. Sadegh Movahed, arXiv:1104.4713

[20] X. Zhang, Phys. Lett. B 648 (2007)1.

[21] J. Zhang, X. Zhang, H. Liu, Eur. Phys. J. C 54 (2008) 303;

J .P Wu, D. Z. Ma, Y. Ling, Phys. Lett. B 663, (2008) 152;

A. Sheykhi, A. Bagheri and M. M. Yazdanpanah, JCAP 09 (2010 ) 017.

[22] S. Tsujikawa, M. Sami, Phys. Lett. B 603 (2004) 113.

[23] B. Wang, Y. Gong and E. Abdalla, Phys. Lett. B 624 (2005) 141;

B. Wang, C. Y. Lin and E. Abdalla, Phys. Lett. B 637 (2005) 357.

[24] D. Pavon, W. Zimdahl, Phys. Lett. B 628 (2005) 206;

N. Banerjee, D. Pavon, Phys. Lett. B 647 (2007) 477.

[25] H. Zhang and Z. Zhu, Phys. Rev. D 73 (2006) 043518. 